\title{
Spatial updating in superimposed real and virtual environments
}

\author{
Xiaoang Irene Wan, Ranxiao Frances Wang, and James A. Crowell \\ University of Illinois at Urbana-Champaign, Urbana, Illinois
}

\begin{abstract}
Wang (2004) showed that people do not always simultaneously update their relationships to real and imagined environments in a dual-environment situation. Employing the same paradigm, we examined whether spatial updating operates on virtual reality as it does on a real or fictitious environment. Participants learned target locations in a real room and a virtual kitchen. Then they turned to face targets either in the room or in the kitchen, while blindfolded, and pointed to the targets before and after turning. Participants kept track of their orientation in both environments equally efficiently, regardless of explicit instructions. In contrast, when the real environment was described verbally but not directly perceived, participants automatically updated the virtual kitchen but not the room. These results suggest that people automatically update a virtual environment as they do a real one when the two environments are superimposed. The automaticity of spatial updating is discussed.
\end{abstract}

Imagine that you stand up from a chair, turn left, and move one step to the right, while the chair remains stationary. Where is the chair relative to your new orientation and location? Certainly you do not think the chair is still behind you; otherwise you will fall to the ground when you try to sit in the chair again. You know the chair now is on your left, because during your movements you keep track of your relationship with the surroundings. This process is known as spatial updating. Spatial updating plays a central role in our interaction with the world, since it allows us to perceive a fairly stable world, to use stable objects as landmarks in our navigation, and to detect location or orientation changes of mobile objects.

Spatial updating is not unique to human beings. For example, insects such as ants and bees can keep track of their current locations, relative to their original locations, by integrating their movement vectors while navigating through an environment, a simple form of spatial updating referred to as path integration. Studies have shown that path integration is common to many species, including insects (Müller \& Wehner, 1988, 1994; Wehner \& Srinivasan, 1981), birds (Regolin, Vallortigara, \& Zanforlin, 1995; von Saint Paul, 1982), and mammals (Etienne, Maurer, \& Seguinot, 1996; Mittelstaedt \& Mittelstaedt, 1980). Interestingly, a study on desert ants (Schmidt, Collett, Dillier, \& Wehner, 1992) indicated that ants keep adding up their homing vectors, even on their returning journey, suggesting that spatial updating occurs all the time to oriented animals.

As for humans, spatial updating uses various types of perceptual cues and can operate in the absence of visual information. Spatial updating is easier for physical movements and more effortful for imagined movements (Eas- ton \& Sholl, 1995; Farrell \& Robertson, 1998; Klatzky, Loomis, Beall, Chance, \& Golledge, 1998; Presson \& Montello, 1994; Rieser, 1989). For example, Rieser showed that blindfolded participants were able to keep track of targets relative to them during their turning or translation. They pointed to the targets more accurately when they actually moved than when they only imagined moving. Results of studies comparing self-rotation and display rotation (Simons \& Wang, 1998; Wang \& Simons, 1999; Wraga, Creem-Regehr, \& Proffitt, 2004), or manipulating the type of movements involved (Klatzky et al., 1998; May \& Klatzky, 2000), suggest that the vestibular and proprioceptive information accompanying physical movement plays an important role in spatial updating.

\section{The Automaticity of Spatial Updating}

Although an increasing number of studies have examined properties of spatial updating, one of its key features - that is, whether spatial updating is automatic or not-remains controversial, both in terms of when spatial updating is automatic and what "automatic updating" is referred to. There are two main lines of research regarding automatic spatial updating.

First, some researchers showed that spatial updating occurs involuntarily and people have difficulty preventing it from happening (Farrell \& Robertson, 1998; Farrell \& Thomson, 1998; May \& Klatzky, 2000). This suggests that spatial updating is "automatic," in the sense that it is difficult to suppress. For example, Farrell and Robertson tested blindfolded participants' memory of targets after they turned their bodies. When participants were asked to ignore their rotations and point to the targets as if they were still facing the original orientation, they had more er-

X. I.Wan, xwan@alumni.illinois.edu 
rors and longer response latencies, indicating that spatial updating is difficult to suppress when observers change locations and orientations. This phenomenon is also referred to as obligatory spatial updating (Riecke, Cunningham, \& Bülthoff, 2007; Riecke, von der Heyde, \& Bülthoff, 2004, 2005).

Contrary to these findings, studies have also shown that spatial updating can be suppressed under certain conditions. The occurrence of spatial updating can be modulated by task requirements, participants' level of awareness, and response modality. Waller, Montello, Richardson, and Hegarty (2002) showed that spatial updating can be undone with little cost to performance. Their participants learned a large four-point path from one position, then judged relative directions as if they were on another position on the path. Before making judgments, participants rotated their bodies $180^{\circ}$. One group of participants was asked to ignore their rotation and another group of participants was instructed to keep track of where the path was, relative to their new orientation. Participants ignoring their rotation showed benefits in both reaction times (RTs) and pointing accuracies, suggesting that certain task requirements and different levels of awareness can make spatial updating easier to undo.

Second, some studies show that spatial updating is "automatic," in the sense that it is effortless and does not require attentional focus. For example, when people are required to point to targets relative to their current position and orientation, their performance is independent of, or only slightly influenced by, the magnitude of prior movement they have made (Farrell \& Robertson, 1998; Rieser, 1989; Wraga et al., 2004). Moreover, spatial updating seems to operate without focused attention. Wang and Brockmole (2003b) located participants in two superimposed environments and examined whether people simultaneously update one environment when they are instructed to update the other (see below for more detailed discussion of this dual-environment paradigm). They found that people kept track of their relationships to the immediate surroundings when they were only instructed to update the remote environment. Wang (2004) also showed that people updated the real environment when they were asked to update only the imagined environment. All these results suggest that spatial updating is relatively effortless and can operate without explicit instruction or attentional focus.

However, there is also evidence to suggest that spatial updating is not always easy to do. Attentional focus and cognitive strategy do influence spatial updating under certain conditions. For example, Amorim, Glasauer, Corpinot, and Berthoz (1997) showed that blindfolded participants estimated a target's orientation more quickly and accurately when they attended to the target itself than when they attended to their path, implying an influence of attentional focus on spatial updating. Moreover, spatial updating does not always simultaneously occur in multiple superimposed environments. Wang and Brockmole (2003b) reported that a more remote environment was not updated when participants were instructed to update their immediate environment. Similarly, an imagined environ- ment was not updated when people updated their relationships to the real environment (Wang, 2004). These findings suggest that the "easy-to-do" side of spatial updating may be modulated by the nature of the surrounding environment and movement conditions. In short, not all types of environments are treated in the same way by the spatial updating system.

These findings also suggest that automatic spatial updating may depend on many factors, such as type of environment, task demand, perceptual and cognitive information available at various stages of the process, and so on. In the present study, we examined when spatial updating is easy to do, focusing on the environment type (virtual reality) and the influence of perceptual information.

\section{Virtual Reality and Spatial Updating}

Virtual displays and virtual environments, also referred to as virtual reality (VR), are increasingly used in research on visual and spatial cognition. VR is a perceptual simulation of reality. Most VR systems emphasize visual simulations; more advanced VR systems also include other senses, such as hearing and touch. Modern VR systems can be divided into simple VR systems, such as desktop VR, and sophisticated VR systems, such as head-mounted display (HMD) and Cave. Both types of VR systems allow users to control their exploration of the virtual environment, but via different types of input. As a research tool, VR has advantages and disadvantages compared with real environments (Loomis, Blascovich, \& Beall, 1999). Although virtual environments often have technological limitations, such as a small field of view, lack of realistic environmental modeling, and slow image generation, using VR allows researchers to study situations that are difficult to control in the real world, to display complex scenes, to control stimuli more accurately, and to manipulate parameters flexibly.

One intriguing characteristic of VR is its dual nature. On the one hand, VR provides sensory stimulation that approximates that of real environments. VR allows people to interact with objects and spatial environments as if they were in a different location and time. For example, an HMD can present a visual scene, play sounds, and update both in response to head movements. These simulations allow the users to experience immersion or presence in VR (Heeter, 1992; Held \& Durlach, 1992; Loomis, 1992; Sheridan, 1992). On the other hand, VR is fictitious at a conceptual level because people usually know it is not real. VR may vividly simulate an environment, yet the users know that is not where their bodies physically are. For example, the visual input of an HMD may simulate a forest, but the users know that they are actually standing in a laboratory. The dual nature of VR raises interesting questions about cognitive processes (e.g., spatial updating and navigation) involving VR, real environments, and fictitious or imaginary environments.

It has been shown that people are able to update their position relative to VR, and spatial updating can sometimes be both easy to do and difficult to suppress in VR (e.g., Riecke et al., 2007; Riecke et al., 2004, 2005; Wraga et al., 2004). However, there are debates about when and 
what kind of information is required for spatial updating to automatically and effectively occur in VR. Several studies have suggested that vestibular and proprioceptive information are important, and that optical flow is not enough (Klatzky et al., 1998). People performed spatial updating better when they actually moved in VR than when they physically remained still and used only optical information from VR (Chance, Gaunet, Beall, \& Loomis, 1998; Pausch, Proffitt, \& Williams, 1997). Self-rotation in VR elicited better spatial updating performance than display rotation did (Wraga et al., 2004). Yet Riecke and colleagues showed that visual information provided by VR is enough to trigger both easy-to-do and difficultto-suppress spatial updating. They emphasized that VR has to simulate a consistent and complete scene in order for visual information to elicit automatic spatial updating without the aid of vestibular and proprioceptive information. To sum up, optical flow alone does not necessarily support automatic spatial updating. Additional body senses, such as information from the vestibular, proprioceptive, and efferent systems, allow observers to better estimate target direction or distance (Bakker, Werkhoven, \& Passenier, 1999; Lathrop \& Kaiser, 2002; Ruddle, Payne, \& Jones, 1999) and to transfer knowledge obtained from VR to the real world (Farrell et al., 2003; Grant \& Magee, 1998).

In previous studies of spatial updating in VR, VR has always been the attentional focus in the spatial updating tasks and the only environment that people needed to update. Little is known about whether people automatically update their relations to VR when they focus on another environment. Previous studies have suggested that attentional focus and the nature of the surrounding environment modulate the automaticity of spatial updating (Amorim et al., 1997; Wang, 2004; Wang \& Brockmole, 2003b), but it is not well understood how attentional focus influences spatial updating in VR. Moreover, most studies focused on different conditions during locomotion (i.e., real, visual vs. imagined locomotion) instead of on the nature of the environments themselves (Klatzky et al., 1998). In other words, few studies have examined how learning different types of environments influences subsequent spatial updating processes. The present article examines this issue using the dual-environment paradigm developed by Wang and colleagues (Wang, 2004; Wang $\&$ Brockmole, 2003b).

\section{The Dual-Environment Paradigm and the Present Study}

Wang and Brockmole (2003b) asked people to learn target locations in two superimposed environments (a lab room and the surrounding campus). Participants were then blindfolded and instructed to make a sequence of turns to face different objects in one of the environments. While they turned, their relationship to both environments changed simultaneously, but only one environment was explicitly updated. The environment with the turning targets was explicitly processed, whereas the other environment was irrelevant to the turning task and became the "background." After turning, participants were asked to point to targets in both environments. People showed impairment for the distant environment when it was not explicitly updated, but showed no such effect for a near "background" environment.

Wang (2004) employed the same paradigm to compare real and imagined environments. After participants learned targets in the experiment room, they were asked to imagine being in their own kitchen and to recall objects in the kitchen. Then they were blindfolded and instructed to rotate relative to either the real room or to the imaginary kitchen. When people rotated their bodies relative to the imagined kitchen, they also automatically updated their relationship to the room without being asked to do so. In contrast, when they turned around in the room, they did not update their relationship to the imagined kitchen. In short, when people are situated in multiple environments, spatial updating operates on real and unreal (fictitious, like pure imagination) environments differently.

In this article, we report two experiments in which we examined how spatial updating operates when observers are placed in superimposed real and virtual environments. We used an HMD to present a virtual kitchen to participants, who were required to learn the locations of targets around them in both the virtual kitchen and the real room, and to move around in these environments. Do participants keep track of their orientations relative to the two environments equally well, when required to explicitly update only one of them? Do participants update their relationship to both environments simultaneously? On the basis of Wang's (2004) findings on the distinction between automatic spatial updating ${ }^{1}$ in real and imagined environments, we examined whether VR invokes the same types of spatial representations and spatial updating process as a real environment, a fictitious environment such as pure imagination, or both.

\section{EXPERIMENT 1}

The purpose of this experiment was to address three questions: (1) Is explicit spatial updating equally efficient in the virtual and real environments? (2) Do people update their spatial relationship to the real environment when they turn around, relative to the virtual environment? (3) Does spatial updating automatically occur in the virtual environment when the real environment is updated explicitly?

\section{Method}

Participants. Sixteen undergraduate students from the University of Illinois at Urbana-Champaign (UIUC) participated in this experiment to fulfill an introductory psychology class requirement. They were randomly assigned to two conditions. Eight participants were assigned to an update virtual environment condition, and the other 8 were assigned to an update real environment condition.

Apparatus. The experiment was conducted in an $8 \times 15 \mathrm{ft}$ rectangular room containing five targets: a door, a closet, a lamp, a VCR, and a poster (see Figure 1A for an illustration). It also contained some other objects necessary to present the virtual kitchen, such as computers and the HMD equipment. A swivel chair was placed at the center of the room. A video camera was mounted directly above the chair pointing downward; its output was visible on a TV screen overlaid with a $360^{\circ}$ protractor for estimating pointing angles during 
A

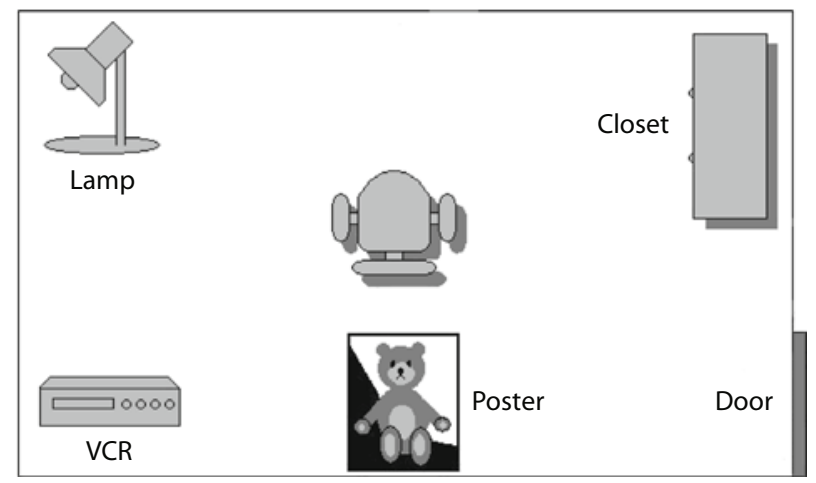

B

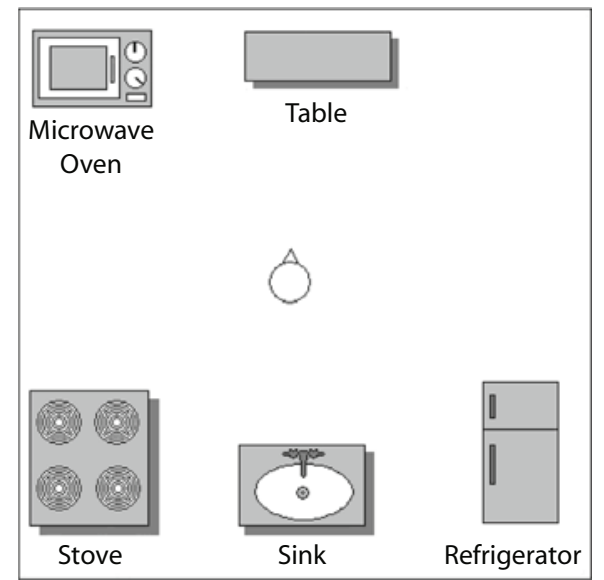

Figure 1. (A) An overhead view of the experiment room with five targets, including the door, closet, lamp, VCR, and poster. A swivel chair was placed at the center of the room, with an overhead video camera mounted above. Nontarget objects are not shown in this figure. (B) An overhead view of the virtual kitchen with five targets, including the microwave oven, stove, sink, refrigerator, and table.

the learning stage and was recorded by a VCR for offline measurement of pointing errors during the main sessions.

A $24 \times 24 \mathrm{ft}$ virtual kitchen was created using Maya, a 3-D modeling software package. As shown in Figure 1B, the virtual kitchen contained five targets: a refrigerator, a table, a microwave oven, a stove, and a sink. It also contained some other objects necessary for an ordinary kitchen, such as stools near the table and cups in the sink. Participants wore a 5DT (Fifth Dimension Technologies) stereoscopic HMD with a Logitech 6-degree-of-freedom head tracker. Two computers were used to drive the stereo 3-D simulation of the virtual kitchen that the HMD provided. The display for each eye had a resolution of 800 (horizontal) $\times 600$ (vertical) pixels. The optical field of view for each eye was $26^{\circ}$ diagonal. Figure 2 shows a screen shot from one eye of the HMD.

Design and Procedure. The experimental design was similar to that of Wang (2004). The whole experiment consisted of five stages. In the first stage, all participants learned the room targets, then were tested; in the second stage, they learned the kitchen targets, then were tested. These two stages were termed before-turning stages. The third stage was a turning stage, in which participants in the update virtual environment condition turned relative to the kitchen targets, whereas those in the update real environment condition ro- tated their bodies relative to the room targets. The fourth and fifth stages were termed after-turning stages. Participants in the update virtual environment condition were tested with the kitchen targets in the fourth stage, then with the room targets in the fifth stage. In contrast, participants in the update real environment condition were tested with the room targets in the fourth stage, then with the kitchen targets in the fifth stage. Each participant finished the experiment individually. Targets were always randomized for each participant for each stage, except for the turning stage.

At the beginning of the first stage, the participant entered the room, sat in the chair facing in a fixed direction, as shown in Figure $1 \mathrm{~A}$, and looked around for as long as was necessary to remember the locations of room targets. Then the participant was asked to close his/her eyes and point to each target. If there were any pointing errors, the participant looked at the targets again. This procedure was repeated until the participant had a pointing error of less than $10^{\circ}$ from the correct direction with eyes closed. Then the participant put on a blindfold and earphones and held a wireless mouse in one hand. Instructions and target names were delivered through the earphones, and static white noise was also played in the earphones to prevent external noise from serving as a directional cue. Object names were read one at a time. Upon hearing each name, the participant physically remained still, pointed toward the named object using whichever hand was convenient, and clicked the wireless mouse as soon as his/her finger was in the desired direction.

At the beginning of the second stage, the participant was instructed to close his/her eyes. Then the experimenter helped the participant put on the HMD, made sure the participant could not see the room at the same time, then asked the participant to open his/her eyes. As before, the participant turned his/her head and learned the virtual target locations with errors within $10^{\circ}$. The experimenter did not give explicit instructions to associate the two environments, or to use any particular strategy. After the learning phase, the experimenter asked the participant to close his/her eyes, helped the participant remove the HMD, then blindfolded the participant. Thus, the participant was not allowed to look at the room again. Then the participant was tested for the virtual targets as the participant had been the case for the real targets. These two learning stages served as a baseline measure of participants' initial memory of the targets in the two environments.

The next stage was the turning stage. While the participant still sat in the chair facing in the fixed direction, the experimenter read the target names from one of the environments in a pseudorandom

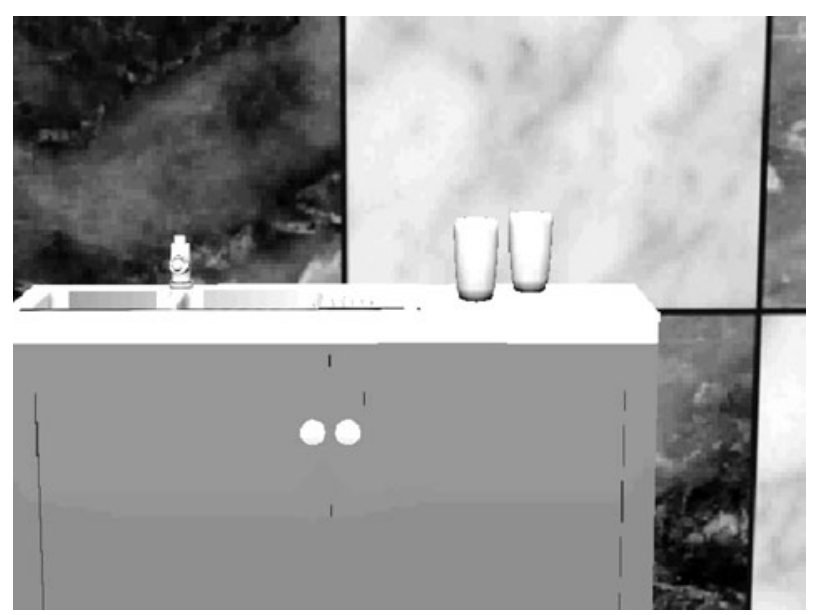

Figure 2. An example of what participants might see when they looked at the sink in the virtual kitchen. The display presented to each eye had a resolution of 800 (horizontal) $\times 600$ (vertical) pixels and subtended $26^{\circ}$ diagonal. 
sequence through the headphones. The participant swiveled his/her chair to face each target, clicked the wireless mouse as soon as his/ her body was in the desired orientation, and stayed in this orientation until the next target name was presented. Participants in the update virtual environment condition turned to face the kitchen targets, and those in the update real environment condition turned to face the room targets. Each participant made a total of five turns, facing each target once. The last target named was always the sink in the update virtual environment condition and the poster in the update real environment condition, and participants remained in this orientation throughout the final testing stage. The other four target names were presented in a random order. Thus, the final facing direction and, consequently, the testing direction in the final stage were roughly matched for the two groups.

Finally, knowledge of the target directions in both environments was tested. The participant remained in the same orientation as at the end of the turning stage and pointed to the targets named by the experimenter. For participants in the update virtual environment condition, kitchen targets were tested before room targets. For participants in the update real environment condition, room targets were tested before kitchen targets. Targets in the two environments were tested consecutively with no break between the environments. Each participant made a total of 10 responses, 5 for each environment.

Data collection and encoding. RTs were recorded for each pointing or facing response. The experimenter simultaneously read the target name and clicked the mouse once to start each trial, and the participant clicked the wireless mouse to end the trial. The RT for each trial was the duration between these two clicks.

Unsigned angular errors were measured after the experiment. When the videotape was played, the experimenter used the $360^{\circ}$ protractor on the TV screen mentioned above to measure the pointing directions and the facing directions. The correct angles for the room targets closet, door, poster, VCR, and lamp were $68^{\circ}, 113^{\circ}$, $200^{\circ}, 239^{\circ}$, and $298^{\circ}$, respectively. The correct angles for the kitchen targets table, refrigerator, sink, stove, and microwave were $15^{\circ}, 125^{\circ}$, $192^{\circ}, 252^{\circ}$, and $310^{\circ}$, respectively. For the pointing responses, the direction of the pointing finger (the index finger for most participants) was recorded as the pointing direction. For the turning responses, the direction perpendicular to the participants' shoulders was recorded as the facing direction. The unsigned angular error was the absolute value of the difference between the correct direction and the pointing or facing direction.

In particular, we took the turning errors into account when we encoded the angular errors of the after-turning pointing directions. For example, suppose that the correct facing direction at the end of the turning stage was $\alpha^{0}$ when participants were asked to face the poster or the sink, but 1 participant turned to face $\alpha+\beta^{\circ}$ and remained in the same orientation during the after-turning stages. In other words, she was physically $\beta^{\circ}$ off the poster or the sink, while thinking she was facing it. Thus, all subsequent pointing responses would be shifted by $\beta$. In this case, when we encoded her after-turning pointing directions, we subtracted the common error $\beta^{\circ}$ from each observed pointing direction to obtain the adjusted pointing direction.

RTs and unsigned angular errors were averaged across all five targets in each environment. Although targets could be classified as stable objects (large and built-in appliances such as doors and stoves) and unstable objects (small and movable appliances such as VCRs and microwaves), Wang (2004) reported no influence of target stability on spatial updating.

\section{Results and Discussion}

To examine whether people explicitly updated the real and virtual environments equally well, we first compared their turning performance in the two environments. Table 1 shows the average angular errors and response latencies of the turning performance of the two groups. There was no significant difference in either angular errors or RTs between the room and kitchen [angular errors, $t(14)=1.54$, $p=.15$; RT, $t(14)=0.21, p=.84]$. Moreover, 2 (stage: before or after turning) $\times 2$ (environment: kitchen targets in the update virtual environment condition or room targets in the update real environment condition) ANOVAs on angular errors and RTs showed only a main effect of stage on angular errors $[F(1,14)=11.27, p<.01]$. No other main effect or interaction was significant $(F \mathrm{~s}<2.5, p \mathrm{~s}>.13)$. These results suggest that explicit updating of the real and virtual environments was equally efficient.

To examine automatic updating of the room, we performed 2 (stage: before or after updating) $\times 2$ (environment: real or virtual) ANOVAs on angular errors and RTs in the update virtual environment condition. The average angular errors and RTs of the pointing performance in the update virtual environment condition are shown in the left panel of Figures 3A and 3B, respectively. The only significant result was the main effect of stage on angular errors $[F(1,7)=7.17, p<.05]$. No other main effects or interactions were significant $(F \mathrm{~s}<2.22, p \mathrm{~s}>.18)$. These results suggest that participants updated the background room as efficiently as the explicitly updated kitchen; that is, they updated the room automatically, while turning relative to the virtual kitchen.

The more interesting question is whether the virtual environment was treated the same as the real environment and thus updated automatically, or whether it was treated as a fictitious, imagined environment and thus not updated automatically. To address this issue, we performed 2 (stage: before or after updating) $\times 2$ (environment: real or virtual) ANOVAs on angular errors and RTs in the update real environment condition. The average angular errors and RTs of the pointing performance in the update real environment condition are shown in the right panel of Figures $3 \mathrm{~A}$ and $3 \mathrm{~B}$, respectively. The main effect of stage was significant on angular errors $[F(1,7)=7.06, p<.05]$, and the main effect of environment was significant on RTs $[F(1,7)=26.84, p<.01]$. No other main effects or in-

Table 1

Mean Angular Errors (in Degrees) and Reaction Times (RTs, in Seconds) in the Turning Stage, With Standard Errors

\begin{tabular}{|c|c|c|c|c|c|c|c|c|}
\hline \multirow[b]{3}{*}{ Turning Stage } & \multicolumn{4}{|c|}{ Experiment 1} & \multicolumn{4}{|c|}{ Experiment 2} \\
\hline & \multicolumn{2}{|c|}{ Angular Errors } & \multicolumn{2}{|c|}{ RTs } & \multicolumn{2}{|c|}{ Angular Errors } & \multicolumn{2}{|c|}{ RTs } \\
\hline & $M$ & $S E$ & $M$ & $S E$ & $M$ & $S E$ & $M$ & $S E$ \\
\hline Update virtual kitchen & 19.58 & 3.06 & 3.88 & 0.60 & 13.50 & 2.05 & 4.20 & 0.43 \\
\hline Update real room & 13.90 & 2.05 & 3.73 & 0.40 & 20.60 & 3.45 & 5.01 & 0.37 \\
\hline
\end{tabular}



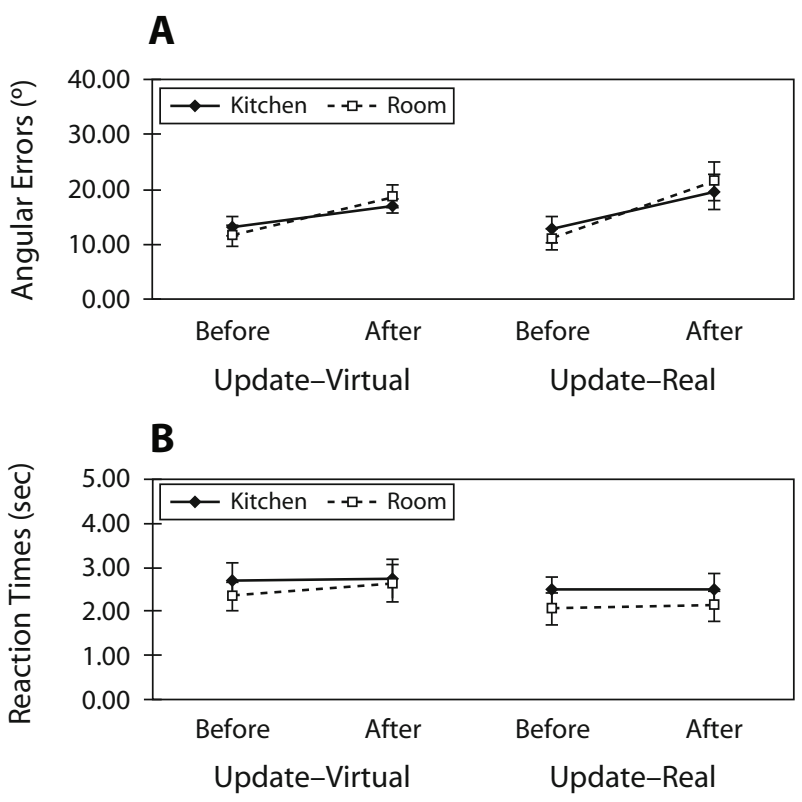

Figure 3. Mean angular errors and reaction times of Experiment 1 in different conditions (update virtual environment or update real environment) and stages (before or after turning). The error bars are standard errors.

teractions were significant $(F \mathrm{~s}<1.46, p \mathrm{~s}>.26)$. These results suggest that this group of participants responded faster overall to room targets than to virtual ones, both before and after the turning stage; yet explicit updating relative to the room during the turning stage caused no more impairment to the background kitchen than to the explicitly updated room. In other words, they automatically updated the virtual environment while explicitly updating the real one.

Before drawing any conclusions, we also ruled out the possibility that the apparent automatic updating of both background environments was an experimental artifact of a target association strategy; that is, four room targets had similar locations with corresponding kitchen targets: lamp $\left(298^{\circ}\right)$ and microwave oven $\left(310^{\circ}\right)$, VCR $\left(239^{\circ}\right)$ and stove $\left(252^{\circ}\right)$, poster $\left(200^{\circ}\right)$ and sink $\left(192^{\circ}\right)$, door $\left(113^{\circ}\right)$ and fridge $\left(125^{\circ}\right)$. The alignment of the targets in the two environments might have led participants to update only the environment they were instructed to, then remember how each background target was located relative to each explicitly updated target. If participants used this target association strategy to make responses in the dualenvironment situation, they would be able to locate the background targets without directly updating them.

We ruled out this possibility by selecting the unaligned targets (the closet in the room and the table in the kitchen) and performing the analyses on the data of these targets only. First, we performed 2 (stage: before or after updating) $\times 2$ (environment: real or virtual) ANOVAs on angular errors and RTs in the update virtual environment condition. The only significant results were the main effects of stage on angular errors $[F(1,7)=16.46, p<.01]$ and RTs $[F(1,7)=10.74, p<.05]$. No other main effects or interactions were significant $(F \mathrm{~s}<2.52, p \mathrm{~s}>.15)$. Second, we performed 2 (stage: before or after updating) $\times 2$ (environment: real or virtual) ANOVAs on angular errors and RTs in the update real environment condition. The main effect of stage was significant on angular errors $[F(1,6)=36.59, p<.01]$, and the main effect of stage was marginally significant on RTs $[F(1,7)=4.00$, $p=.086]$. None of the other main effects or interaction effects was significant $(F \mathrm{~s}<1.14, p \mathrm{~s}>.32)$. These results are consistent with the findings when both aligned and unaligned targets are included in the analyses. In addition, although four pairs of targets were close to each other, it should be noted that there are still $8^{\circ}-13^{\circ}$ difference between the two targets of each pair. If the participants only updated the relationship to one target and used the same direction for the other target, this usage of the target association strategy should introduce greater errors to the "background" targets, which were not shown in the data. All these results rule out the possibility that participants used the target association strategy to equally update both environments.

In summary, results of the turning stage suggest that people had equivalent performance in updating the virtual and real environments when explicitly instructed to do so. Also, results of the before- and after-turning stages suggest that they automatically updated either environment while explicitly updating the other. In both updating conditions they pointed less accurately after the turning stage, but this updating cost did not differ between the two environments according to the updating instructions.

However, there is one potential limitation with this experiment. That is, we obtained null results, and a lack of difference between the two types of environments is often difficult to interpret. Thus, we further examined whether the spatial updating in VR is automatic by comparing the virtual kitchen with an environment not updated automatically. Wang (2004) showed that automatic spatial updating did not occur for a verbally described environment when participants were explicitly instructed to update their relation to an imagined environment. In Experiment 2, we situated participants in a verbally described room and a virtual kitchen to examine whether a virtual environment and a verbally described environment are updated differently.

\section{EXPERIMENT 2}

\section{Method}

Sixteen UIUC undergraduate students participated in this experiment to fulfill an introductory psychology class requirement. None of them had participated in Experiment 1. They were randomly assigned to two conditions. Eight participants were assigned to the update virtual environment condition, and the other 8 were assigned to the update real environment condition. Apparatus, design, and data collection were the same as in Experiment 1, except that participants never saw the experiment room during this experiment, and were blindfolded before entering the room. The blindfolded participant was brought to the center of the room, then sat in the chair. The experimenter described the names of the five room targets, and moved the participant's arm to point to each target while it was named. As before, the participant was asked to point to each target, and the 
experimenter corrected any errors in the pointing responses. This learning procedure was repeated until the participant's pointing was within $10^{\circ}$ of the correct direction, then the real test started. The remaining procedure was exactly the same as that in Experiment 1. When learning virtual kitchen targets, participants wore the HMD and viewed the virtual kitchen, as was done in Experiment 1.

We use "verbally described" to specify the nature of the room in this experiment as shorthand for "communicated by the experimenter, as opposed to directly perceived." When the experimenter named each target and moved the participant's arm to point to the target, the participant had some proprioceptive information, but it was communicated by the experimenter. It should also be noted that only the name of each target was described, whereas neither distance nor azimuth was provided. Thus, the "verbally described" room used in this experiment and by Wang (2004) is different from other narrative-described environments in the literature (e.g., Avraamides, 2003; de Vega \& Rodrigo, 2001).

\section{Results and Discussion}

The average angular errors and RTs of the turning performance of the two groups are shown in Table 1. We conducted two analyses to examine whether people explicitly updated the verbally described room and virtual kitchen equally well. First, we compared turning performance in the two environments. There was no significant difference in angular errors or RTs between environments [angular errors, $t(14)=1.77, p=.098$; RTs, $t(14)=1.44, p=$ .17]. Second, we performed 2 (stage: before or after turning) $\times 2$ (environment: kitchen targets in the update virtual environment condition or room targets in the update real environment condition) ANOVAs on angular errors and RTs. The main effect of stage was significant on angular errors $[F(1,14)=7.35, p<.05]$, and the interaction between stage and environment was also significant on angular errors $[F(1,14)=6.04, p<.05]$. These results

A

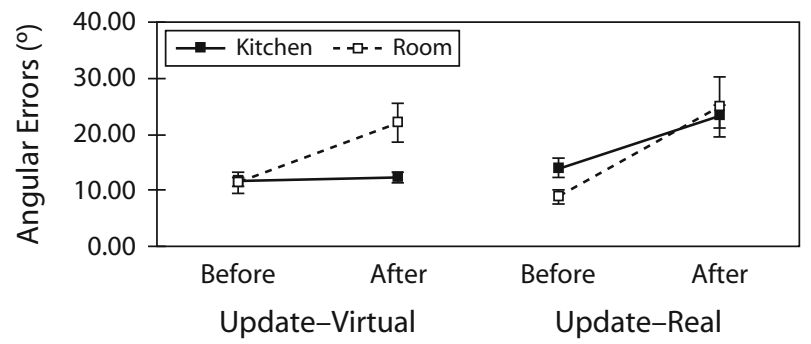

B

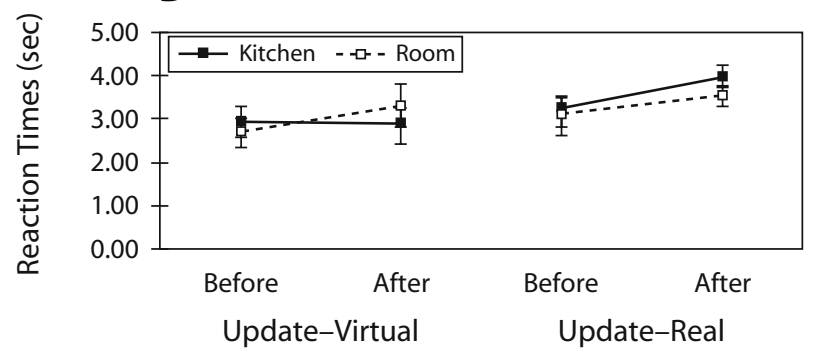

Figure 4. Mean angular errors and reaction times of Experiment 2 in different conditions (update virtual environment or update real environment) and stages (before or after turning). The error bars are standard errors. suggest that participants explicitly updated the virtual environment slightly better than they did the verbally described environment.

To examine automatic updating of the verbally described room, we performed 2 (stage: before or after updating) $\times 2$ (environment: real or virtual) ANOVAs on angular errors and RTs in the update virtual environment condition. The average angular errors and RTs of the pointing performance in the update virtual environment condition are shown in the left panel of Figures 4A and 4B, respectively. The main effect of stage was significant for both angular $[F(1,7)=7.53, p<.05]$ and RTs $[F(1,7)=6.97, p<.05]$. Most interestingly, the interaction between stage and environment was also significant for both angular errors $[F(1,7)=7.28, p<.05]$ and RTs $[F(1,7)=6.82, p<.05]$. Thus, performance was significantly more impaired for the verbally described room in the background than for the virtual kitchen in the foreground, suggesting that participants did not automatically update the verbally described room while explicitly updating the virtual kitchen.

Similarly, we performed 2 (stage: before or after updating) $\times 2$ (environment: real or virtual) ANOVAs on errors and RTs in the update real environment condition to examine automatic updating of the virtual kitchen. The average angular errors and RTs of the pointing performance in the update real environment condition are shown in the right panel of Figures $4 \mathrm{~A}$ and $4 \mathrm{~B}$, respectively. The results showed main effects of stage for both angular errors $[F(1,7)=11.28, p<.05]$ and $\operatorname{RTs}[F(1,7)=5.81$, $p<.05]$. Yet there were neither main effects of environment nor significant interactions between environment and stage $\left(F_{\mathrm{S}}<1.74, p \mathrm{~s}>.22\right)$. Thus, participants automatically updated the virtual environment while explicitly updating the verbally described room.

In summary, participants showed slightly better turning performance for the virtual kitchen than for the room, updating the virtual environment slightly better than they did the verbally described one when they were instructed to do so. Moreover, participants automatically updated the virtual kitchen when it was in the background but did not do so for the verbally described room. In other words, the spatial updating system treated the visible virtual environment in the same way in which it processed the visible real environment, whereas it treated the invisible and verbally described environment as it processed the fictitious environments, such as imagination. These results suggest that the automatic updating of the virtual kitchen is not a result of insensitivity of the methodology.

\section{GENERAL DISCUSSION}

We compared spatial updating in real and virtual environments in two experiments using the dual-environment paradigm. Participants learned targets in a real room and a virtual kitchen. They then turned to face a series of targets, either in the room or in the kitchen, while blindfolded. When targets in the room and in the kitchen were visible during the learning stages, participants automatically updated either background environment while explicitly 
updating the other. Specifically, although automatic updating of two environments at the same time might increase the difficulty of updating either one, spatial updating of either environment was not influenced by people's attentional focus, suggesting that superimposed virtual and real environments are updated simultaneously. In contrast, when the real environment was described verbally with experimenter-provided proprioceptive feedback, but was never perceived directly during the learning stages, participants automatically updated the background virtual kitchen but not the background room, suggesting that spatial updating of a verbally described environment is influenced by people's attentional focus. Thus, VR was always automatically updated in the dual-environment paradigm, regardless of how the other environment was learned, suggesting that the spatial updating system treats VR as if it were a directly perceived real environment.

The present study shows that automatic spatial updating might be determined by perceptual experience rather than knowledge. VR is perceptually "real" but conceptually "fictitious." If the spatial updating system treats VR as a fictitious environment, this suggests that top-down information might play a more important role than bottom-up information in the mechanism of automatic spatial updating. Past research of spatial memory showed that knowing the category of an object influences judgment of its location (e.g., Hirtle \& Jonides, 1985; McNamara, 1986; Stevens \& Coupe, 1978), suggesting the influence of topdown information on spatial representation. Thus, it is reasonable to hypothesize that knowing that an environment is real determines whether people automatically update the environment they see. On the contrary, our findings suggest that VR is updated more efficiently than a real environment for which perceptual information is missing. Participants in Experiment 2 accepted the authenticity of the verbal description of the unseen room and realized that the virtual kitchen that they did see was unreal. However, their spatial updating systems still automatically processed the virtual kitchen but not the verbally described real environment. These results suggest that VR involves the same type of representations and cognitive processes as does the real environment in a spatial updating task. This finding is consistent with recent illustration that virtual and real environments elicit similar spatial learning patterns and representations (Williams, Narasimham, Westerman, Rieser, \& Bodenheimer, 2007). To sum up, although top-down information might influence spatial representation and memory in certain tasks, the present study suggests the importance of bottom-up information on automatic spatial updating.

The VR system used in the present research provides reasonable image quality and tracking accuracy. However, the field of view is limited ( $26^{\circ}$ diagonal), and the virtual kitchen is graphically detailed but not very realistic. Despite these limitations, people automatically updated the virtual environment as a real one, even though they were consciously aware that these kitchen objects did not actually exist. Of course, not all VR systems are equivalent; they vary tremendously in image quality, resolution, field of view, refresh rate, simulation lag (the delay between an observer's action and the update of the display), tracking error (the discrepancy between the actual and simulated viewpoints), and so on. VR systems also differ in their input methods (for example, keyboard vs. joystick) and in the mobility of the user (stationary, as in desktop VR, or mobile in HMD and CAVE systems). All these factors may affect the realism of the simulation; thus, generalization of findings from the present study to different types of VR systems should be done cautiously. The present study confirms that HMD VR with limited FOV is good enough to elicit easy-to-do spatial updating which does not require focused attention. This finding confirms the promising future of using HMD VR to help us better understand navigation and spatial reasoning in the real world.

Results of both experiments are consistent with previous findings that spatial updating occurs in VR (Riecke et al., 2007; Riecke et al., 2004, 2005; Wraga et al., 2004). Experiment 2 also confirms previous research that people do not automatically update verbally described environments, and do not necessarily update multiple environments simultaneously, even when they occupy the same physical space (Wang, 2004). These findings are consistent with the proposal that spatial updating is capacity limited (Wang et al., 2006), and different environments have independent representations (Wang \& Brockmole, 2003a).

Our results provide further support on the notion that spatial updating in VR is effortless and easy to do (Wraga et al., 2004). In our experiments, spatial updating operated in VR without observers' focused attention. Participants in the update real environment condition were instructed to update their relationship to the room during the turning stage. When rotating their bodies to face each room target, participants attended to the room and focused on accomplishing their turning relative to the room. Their pointing performance for the after-turning tests revealed that they simultaneously updated the virtual kitchen when explicitly updating the room. In short, spatial updating automatically operates on VR, regardless of attentional focus.

These results are in line with the hypothesis that visual information plays an important role in spatial updating (e.g., Riecke et al., 2007). In both experiments, participants automatically updated the virtual kitchen when it was in the background, suggesting that a fictitious environment can be treated by the spatial updating system like a real environment when direct visual stimulation is provided. Thus, visual information alone is capable of supporting automatic spatial updating. On the other hand, Wang (2004) showed that people automatically update an unseen but touched real environment, suggesting that visual input is not necessary for automatic spatial updating. Moreover, visually learned large environments are not automatically updated (Wang \& Brockmole, 2003b), suggesting that direct visual input during learning is not sufficient for automatic spatial updating.

In comparison with the virtual environment, the verbally described environment in Experiment 2 provided limited spatial information which might hinder the participants in actively or freely apprehending the targets or the spatial structure of the environment. Thus, the reason the verbally described room was not automatically updated might be the absence of perceptual information, the lack 
of information about spatial structure, or both. Nevertheless, environments well learned from full information of everyday experience are not always automatically updated (Wang \& Brockmole, 2003b), suggesting that richness in spatial information is not sufficient for automatic spatial updating. To sum up, two important factors might determine the automaticity of spatial updating: the nature of the environment (e.g., scale), and the way the environment is learned (e.g., availability of perceptual information).

\section{AUTHOR NOTE}

We thank Lance Chong for creating the virtual kitchen and Bradley Nauman for running experiments. We also thank Hank Kaczmarski, director of Integrated Systems Laboratory at UIUC. This research was supported by NSF Grant BCS 03-17681 to R.F.W. Some of the data were presented at the 46th Annual Meeting of the Psychonomic Society, Toronto, 2005. Correspondence concerning this article should be addressed to X. I. Wan, Department of Psychology, University of Illinois at Urbana-Champaign, 603 East Daniel Street, Champaign, IL 61820 (e-mail: xwan@alumni.illinois.edu).

\section{REFERENCES}

Amorim, M.-A., Glasauer, S., Corpinot, K., \& Berthoz, A. (1997) Updating an object's orientation and location during nonvisual navigation: A comparison between two processing modes. Perception \& Psychophysics, 59, 404-418.

AvraAmides, M. N. (2003). Spatial updating of environments described in texts. Cognitive Psychology, 47, 402-431.

Bakker, N. H., Werkhoven, P. J., \& Passenier, P. O. (1999). The effects of proprioceptive and visual feedback on geographical orientation in virtual environments. Presence: Teleoperators \& Virtual Environments, 8, 36-53.

Chance, S. S., Gaunet, F., Beall, A. C., \& Loomis, J. M. (1998). Locomotion mode affects the updating of objects encountered during travel: The contribution of vestibular and proprioceptive inputs to path integration. Presence: Teleoperators \& Virtual Environments, 7, 168-178.

DE VEGA, M., \& Rodrigo, M. J. (2001). Updating spatial layouts mediated by pointing and labeling under physical and imaginary rotation. European Journal of Cognitive Psychology, 13, 369-393.

EAston, R. D., \& Sholl, M. J. (1995). Object-array structure, frames of reference, and retrieval of spatial knowledge. Journal of Experimental Psychology: Learning, Memory, \& Cognition, 21, 483-500.

Etienne, A. S., Maurer, R., \& Seguinot, V. (1996). Path integration in mammals and its interaction with visual landmarks. Journal of Experimental Biology, 199, 201-209.

Farrell, M. J., Arnold, P., Pettifer, S., Adams, J., Graham, T., \& MacManamon, M. (2003). Transfer of route learning from virtual to real environments. Journal of Experimental Psychology: Applied, 9, 219-227.

FARrell, M. J., \& Robertson, I. H. (1998). Mental rotation and automatic updating of body-centered spatial relationships. Journal of Experimental Psychology: Learning, Memory, \& Cognition, 24, 227-233.

FArrell, M. J., \& Thomson, J. A. (1998). Automatic spatial updating during locomotion without vision. Quarterly Journal of Experimental Psychology, 51A, 637-654.

Grant, S. C., \& Magee, L. E. (1998). Contributions of proprioception to navigation in virtual environments. Human Factors, 40, 489-497.

HeEter, C. (1992). Being there: The subjective experience of presence. Presence: Teleoperators \& Virtual Environments, 1, 262-271.

Held, R. M., \& Durlach, N. I. (1992). Telepresence. Presence: Teleoperators \& Virtual Environments, 1, 109-112.

Hirtle, S. C., \& Jonides, J. (1985). Evidence of hierarchies in cognitive maps. Memory \& Cognition, 13, 208-217.

Klatzky, R. L., Loomis, J. M., Beall, A. C., Chance, S. S., \& Golledge, R. G. (1998). Spatial updating of self-position and orientation during real, imagined, and virtual locomotion. Psychological Science, 9, 293-298.

Lathrop, W. B., \& Kaiser, M. K. (2002). Perceiving orientation in physical and virtual environments: Changes in perceived orientation as a function of idiothetic information available. Presence: Teleoperators \& Virtual Environments, 11, 19-32.

Loomis, J. M. (1992). Distal attribution and presence. Presence: Teleoperators \& Virtual Environments, 1, 113-119.

Loomis, J. M., Blascovich, J. J., \& BeALL, A. C. (1999). Immersive virtual environment technology as a basic research tool in psychology. Behavior Research Methods, Instruments, \& Computers, 31, 557-564.

MAY, M., \& KLATZKY, R. L. (2000). Path integration while ignoring irrelevant movement. Journal of Experimental Psychology: Learning, Memory, \& Cognition, 26, 169-186.

McNamara, T. P. (1986). Mental representations of spatial relations. Cognitive Psychology, 18, 87-121.

Mittelstaedt, M.-L., \& Mittelstaedt, H. (1980). Homing by path integration in a mammal. Naturwissenschaften, 67, 566-567.

Müller, M., \& Wehner, R. (1988). Path integration in desert ants, Cataglyphis fortis. Proceedings of the National Academy of Sciences, 85, 5287-5290.

Müller, M., \& Wehner, R. (1994). The hidden spiral: Systematic search and path integration in desert ants, Cataglyphis fortis. Journal of Comparative Physiology A, 175, 525-530.

Pausch, R., Proffitt, D., \& Williams, G. (1997). Quantifying immersion in virtual reality. In Proceedings of the 24th Annual Conference on Computer Graphics and Interactive Techniques (pp. 13-18). New York: ACM Press/Addison-Wesley.

Presson, C. C., \& Montello, D. R. (1994). Updating after rotation and translational body movements: Coordinate structure of perspective space. Perception, 23, 1447-1455.

Regolin, L., Vallortigara, G., \& Zanforlin, M. (1995). Detour behaviour in the domestic chick: Searching for a disappearing prey or a disappearing social partner. Animal Behaviour, 50, 203-211.

Riecke, B. E., Cunningham, D. W., \& Bülthoff, H. H. (2007). Spatial updating in virtual reality: The sufficiency of visual information. Psychological Research, 71, 298-313.

Riecke, B. E., von der Heyde, M., \& Bülthoff, H. H. (2004). Spatial updating in real and virtual environments: Contribution and interaction of visual and vestibular cues. In Proceedings of the 1st Symposium on Applied Perception in Graphics and Visualization (pp. 9-17). New York: ACM Press.

Riecke, B. E., von der Heyde, M., \& Bülthoff, H. H. (2005). Visual cues can be sufficient for triggering automatic, reflex-like spatial updating. ACM Transactions on Applied Perception, 2, 183-215.

RIESER, J. J. (1989). Access to knowledge of spatial structure at novel points of observation. Journal of Experimental Psychology: Learning, Memory, \& Cognition, 15, 1157-1165.

Ruddle, R. A., PAyne, S. J., \& Jones, D. M. (1999). Navigating largescale virtual environments: What differences occur between helmetmounted and desk-top displays? Presence: Teleoperators \& Virtual Environments, 8, 157-168.

Schmidt, I., Collett, T. S., Dillier, F.-X., \& Wehner, R. (1992). How desert ants cope with enforced detours on their way home. Journal of Comparative Physiology A, 171, 285-288.

SheridAn, T. B. (1992). Musings on telepresence and virtual presence. Presence: Teleoperators \& Virtual Environments, 1, 120-126.

Simons, D. J., \& WANG, R. F. (1998). Perceiving real-world viewpoint changes. Psychological Science, 9, 315-320.

Stevens, A., \& Coupe, P. (1978). Distortions in judged spatial relations. Cognitive Psychology, 10, 422-437.

von SAINT PAUl, U. (1982). Do geese use path integration for walking home? In F. Papi \& H. G. Wallraff (Eds.), Avian navigation (pp. 298307). New York: Springer.

Waller, D., Montello, D. R., Richardson, A. E., \& Hegarty, M. (2002). Orientation specificity and spatial updating of memories for layouts. Journal of Experimental Psychology: Learning, Memory, \& Cognition, 28, 1051-1063.

WANG, R. F. (2004). Between reality and imagination: When is spatial updating automatic? Perception \& Psychophysics, 66, 68-76.

WANG, R. F., \& BRockmole, J. R. (2003a). Human navigation in nested environments. Journal of Experimental Psychology: Learning, Memory, \& Cognition, 29, 398-404.

Wang, R. F., \& Brockmole, J. R. (2003b). Simultaneous spatial updating in nested environments. Psychonomic Bulletin \& Review, 10, 981-986. 
Wang, R. F., Crowell, J. A., Simons, D. J., Irwin, D. E., Kramer, A. F., Ambinder, M. S., ET AL. (2006). Spatial updating relies on an egocentric representation of space: Effects of the number of objects. Psychonomic Bulletin \& Review, 13, 281-286.

WANG, R. F., \& Simons, D. J. (1999). Active and passive scene recognition across views. Cognition, 70, 191-210.

WeHNER, R., \& SRINIVASAN, M. V. (1981). Searching behavior of desert ants, genus Cataglyphis (Formicidae, Hymenoptera). Journal of Comparative Physiology, 142, 315-338.

Williams, B., Narasimham, G., Westerman, C., Rieser, J., \& BodenHEIMER, B. (2007). Functional similarities in spatial representations between real and virtual environments. ACM Transactions on Applied Perception, 4, 1-22.
Wraga, M., Creem-Regehr, S. H., \& Proffitt, D. R. (2004). Spatial updating of virtual displays during self- and display rotation. Memory \& Cognition, 32, 399-415.

\section{NOTE}

1. We use the phrase "automatic spatial updating," which emphasizes that spatial updating is "easy to do" without much attentional focus.

(Manuscript received March 18, 2008; revision accepted for publication July 1, 2008.) 